\title{
Modèle d'écoulement bidimensionnel à deux fluides
}

\author{
Two-dimensional, two-fluid flow model
}

\author{
Ph. Gully et F. Werkoff
}

CEA/Service des Transferts Thermiques/CEN-Grenoble

\begin{tabular}{|c|c|}
\hline \multicolumn{2}{|c|}{ Lettres latines } \\
\hline$a$ & : Vitesse du son \\
\hline E & $\begin{array}{l}\text { : Energie transférée à l'interface par unité de } \\
\text { volume et de temps }\end{array}$ \\
\hline$g$ & : Vecteur accélération de la pesanteur \\
\hline$h$ & : Enthalpie massique \\
\hline$J$ & : Débit massique par unité de volume \\
\hline$M$ & $\begin{array}{l}\text { : Quantité de mouvement transférée à l'interface } \\
\text { par unité de volume et de temps }\end{array}$ \\
\hline$P$ & : Pression \\
\hline$r$ & : Variable d'espace; rayon \\
\hline$T$ & : Temps d'intégration \\
\hline$t$ & : Variable de temps \\
\hline$u$ & : Vitesse radiale \\
\hline$V$ & : Vecteur vitesse \\
\hline$w$ & : Vitesse axiale \\
\hline$x$ & : Variable d'espace \\
\hline$z$ & : Variable d'espace \\
\hline \multicolumn{2}{|c|}{ Lettres grecques } \\
\hline$\alpha:$ & Taux de présence local \\
\hline$\Gamma:$ & $\begin{array}{l}\text { Masse transférée à l'interface par unité de volume } \\
\text { et de temps }\end{array}$ \\
\hline$\Delta t$ & : Pas de temps \\
\hline$\delta:$ & Epaisseur du downcomer \\
\hline $\begin{array}{l}\delta h \\
\delta p\end{array}$ & - Ecarts de $h, p$ et $w$ entre deux itérations successives \\
\hline$\delta w$ & \\
\hline$\rho:$ & Masse volumique \\
\hline \multicolumn{2}{|c|}{ Indices } \\
\hline$h:$ & A enthalpie constante \\
\hline$i:$ & Relatif au point $\mathrm{i}$ \\
\hline inj : & Relatif à une quantité injectée \\
\hline J : & Relatif au point $j+1 / 2$ ou $j-1 / 2$ \\
\hline
\end{tabular}

$\begin{array}{ll}j: & \text { Relatif au point } j \\ l: & \text { Relatif à la phase liquide } \\ m: & \text { Indice d'itération } \\ n: & \text { Relatif à l'instant } n \\ p: & \text { A pression contante } \\ r: & \text { En projection sur } r \\ v: & \text { Relatif à la phase vapeur } \\ z: & \text { En projection sur } z\end{array}$

La modélisation des écoulements a subi une évolution considérable ces dernières années, avec la naissance de nombreux codes monodimensionnels permettant la prédition du comportement des réacteurs à eau légère en situations accidentelles. Cependant le caractère complexe de la géométrie de certains composants du réacteur a conduit à développer, pour affiner la prédiction, la modélisation d'écoulements bidimensionnels. Nous présentons un modèle numérique d'écoulement bidimensionnel à deux fluides applicables à différentes configurations d'écoulement. Le système d'équations de ce modèle est discrétisé suivant une méthode semi-implicite à mailles décalées. Nous présentons dans ce qui suit le modèle ainsi que quelques résultats de calcul dans diverses géométries.

\subsection{Système d'équations}

Les équations de base qui permettent la description d'un écoulement diphasique traduisent les trois principes fon- 
damentaux de conservation. Une façon rigoureuse de procéder consiste à écrire les principes de conservation sous forme intégrale (Delhaye, 1970). Ces principes sont écrits pour un volume matériel quelconque composé des deux phases. On suppose que les interfaces n'ont pas d'épaisseur et que la tension superficielle joue un rôle négligeable. L'application de la règle de Leibniz et du théorème de Gauss à ces bilans fait apparaître les termes de transfert de masse, de quantité de mouvement et d'énergie à l'interface. Les équations locales instantanées ainsi obtenues sont rigoureuses et ne font appel à aucune hypothèse particulière. Cependant, le caractère aléatoire de l'écoulement oblige à moyenner les équations de bilan. Dans notre cas, pour résoudre un problème bidimensionnel transitoire, les équations locales instantanées sont moyenneés dans le temps sur un intervalle de temps [ $t-T / 2, t+T / 2$ ]. Le temps d'intégration $T$ est choisi en fonction de critères bien définis. Par exemple, pour un écoulement turbulent monophasique, le temps $T$ doit être choisi suffisamment grand comparé aux fluctuations turbulentes et suffisamment petit comparé à l'échelle de temps de l'évolution des paramètres de l'écoulement. En écoulement diphasique, ce choix n'est pas toujours possible et une discussion complète de ce problème complexe est proposée par Delhaye et Achard (1977 et 1978) qui établissent des critères de choix de ce temps $T$ basés sur l'analyse spectrale du signal mesuré.

Pour obtenir le système d'équations du modèle JEHOVAH un certain nombre d'hypothèses ont été avancées et discutées dans (Gully, 1979). Le système d'équations final est porté en annexe et se compose de :

-2 équations de conservation de la masse relatives aux phases liquide et vapeur;

- 4 équations de conservation de la quantité de mouvement relatives à chaque phase et projetées sur deux directions de l'espace;

- 2 équations de conservation de l'énergie relatives à chaque phase.

Aux seconds membres de ces équations apparaissent les termes de transfert à l'interface $\Gamma, M_{n}, M_{z}$ et $E$ qui représentent le couplage des équations entre elles. Les lois de transferts à l'interface sont définies à partir de corrélations empiriques. Certaines d'entre elles sont exposées dans (Houdayer et al., 1982). Les corrélations introduites dans notre modèle résultent d'une extension de ces lois au cas bidimensionnel.

\subsection{Discrétisation du système d'équations}

La méthode numérique utilisée dans JEHOVAH est la méthode ICE semi-implicite employée également dans le code bidimensionnel BEACON (Broadus et al., 1977). Dans ce paragraphe nous présentons son principe en l'appliquant à un système d'équations particulièrement simple : celui d'un écoulement monophasique et isentropique dans une conduite de section constante.

Avec les inconnues $p, h$ et $w$, le système d'équations s'écrit :

$$
\begin{gathered}
\frac{\partial}{\partial t} \rho+\frac{\partial}{\partial z} \rho w=0 \\
\rho \frac{\partial}{\partial t} w+\rho w \frac{\partial w}{\partial z}+\frac{\partial p}{\partial z}=0
\end{gathered}
$$

$$
\begin{gathered}
\rho \frac{\partial}{\partial t} h+\rho w \frac{\partial h}{\partial z}-\frac{\partial p}{\partial t}-w \frac{\partial}{\partial z} p=0 \\
\rho=\rho(h, p)
\end{gathered}
$$

La méthode consiste notamment en l'utilisation des mailles décalées. Les variables $p$ et $h$ sont définies aux nœuds du maillage (point $j$ ) tandis que $w$ est définie aux milieux des mailles (point $j+1 / 2$ ).

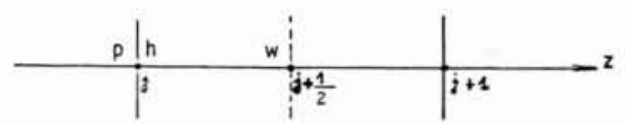

La discrétisation s'effectue sous la forme suivante. On note, par exemple, $p_{j}^{n}$ la valeur de la pression au point $j$ et à l'instant $n$.

$$
\begin{array}{r}
\frac{\rho_{i}^{n+1}-\rho_{i}^{n}}{\Delta t}+\frac{\rho_{j+1 / 2}^{n} w_{j+1 / 2}^{n+1}-\rho_{j-1 / 2}^{n} w_{j-1 / 2}^{n+1}}{\Delta z}=0 \\
\rho_{j+1 / 2}^{n} \frac{w_{j+1 / 2}^{n+1}-w_{j+1 / 2}^{n}}{\Delta t} \\
+\rho_{j+1 / 2}^{n} w_{j+1 / 2}^{n} \frac{w_{j+1}^{n}-w_{j}^{n}}{\Delta z}+\frac{p_{j+1}^{n+1}-p_{j}^{n+1}}{\Delta z}=0 \\
\rho_{j}^{n} \frac{\mathbf{h}_{j}^{n+1}-h_{j}^{n}}{\Delta t}+\rho_{j}^{n} w_{j}^{n} \frac{h_{j+1 / 2}^{n}-h_{j-1 / 2}^{n}}{\Delta z} \\
-\frac{p_{i j}^{n+1}-p_{j}^{n}-w_{j}^{n} \frac{P_{j+1 / 2}^{n}-p_{j-1 / 2}^{n}}{\Delta t}=0}{\Delta z}
\end{array}
$$

On remarque que les équations de masse (1) et d'énergie (3) sont discrétisées au point $j$, tandis que l'équation de moment (2) l'est au point $j+1 / 2$. Toutes les dérivées spatiales sont exprimées à l'aide du schéma amont qui est stable. C'est ainsi que l'indice $J$ est remplacé par $j-1 / 2$ si $w_{j+1 / 2}$ est positif.

Pour résoudre un tel système (équations 1 à 3 ) aux inconnues $p_{j}^{n+1}, h_{j}^{n+1}$ et $\boldsymbol{w}_{j+1 / 2}^{n+1}$, on utilise une méthode itérative de linéaration (méthode de Newton). Soit ${ }^{m} p_{j}^{n+1}$ la valeur de $p_{j}^{n+1}$ à l'itération $m$, pour trouver ${ }^{m+1} p_{j}^{n+1}$ on pose

$$
{ }^{m+1} p_{j}^{n+1}={ }^{m} p_{j}^{n+1}+\delta p_{j}
$$

En opérant de la même manière avec $h_{j}^{n+1}$ et $w_{j+1 / 2}^{n+1}$, on obtient alors un système linéaire de ces trois équations en $\delta p_{j}, \delta h_{j}$ et $\delta w_{j+1 / 2}$. Une combinaison linéaire de ces trois équations conduit à une équation en $\delta p$ uniquement qui s'écrit

$$
-\delta p_{j-1}+\left[2+\left(\frac{\Delta z}{a \Delta t}\right)^{2}\right] \delta p_{j}-\delta p_{j+1}=S
$$

$S$ représente un terme source connu et a la vitesse sonique définie par l'équation

$$
\frac{1}{a^{2}}=\left(\frac{\partial \rho}{\partial p}\right)_{h}+\frac{1}{\rho}\left(\frac{\partial \rho}{\partial h}\right)_{\rho}
$$

L'équation (4) relie entre elles les pressions de trois mailles voisines et l'écriture de celle-ci en tout point du maillage conduit à un système linéaire du type $A \delta P=B$ où $A$ est une matrice tridiagonale, irréductible, à diagonale dominante. 
Il faut remarquer que l'intérêt essentiel de cette méthode consiste en la possibilité d'éliminer les vitesses $w$ et les enthalpies $h$ pour obtenir une équation en pression uniquement. Cette opération est rendue très simple par la prise en compte sous forme explicite des termes convectifs des équations de quantité de mouvement et d'énergie. Ce fait permet en même temps une résolution numérique particulièrement précise des termes convectifs mais impose en revanche que la condition de Courant $(w \Delta t / \Delta z \leqslant 1)$ soit vérifiée. L'équation (4) montre notamment que le couplage entre la pression du point $j$ et les deux pressions voisines est faible, particulièrement pour les fortes vitesses. La matrice A du système linéaire est donc à diagonale fortement dominante. Il en résulte une convergence rapide vers la solution.

Pour notre modèle d'écoulement bidimensionnel à deux fluides, la discrétisation ést effectuée de la même façon en explicitant notamment les termes convectifs des équations de quantité de mouvement et d'énergie (Mazille, 1980). La pression, le taux de présence et les enthalpies de chaque phase sont définis au point $(i, j)$. En revanche, les vitesses radiales et axiales de chacune des phases sont définies respectivement aux points $(i+1 / 2, j)$ et $(i, j+1 / 2)$.

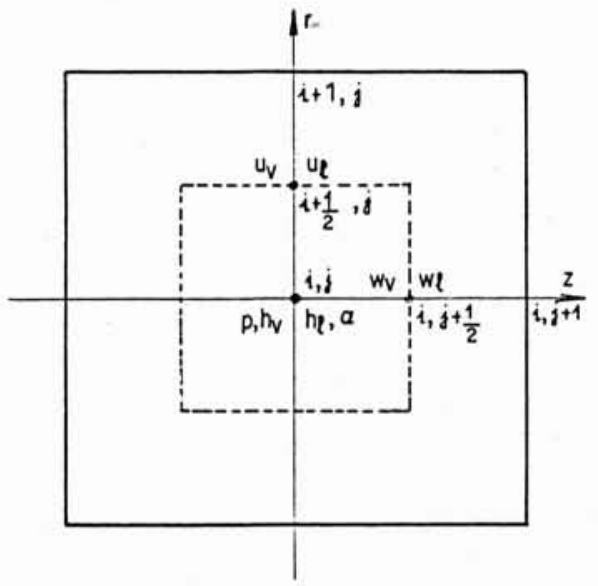

En raison de leur importance primordiale, les termes de transferts à l'interface, qui ne font pas intervenir d'expressions différentielles, sont implicites. Ecrite en tout point du maillage, l'équation aux accroissements de pression conduit à un système linéaire dont la matrice est pentadiagonale.

\section{Application du code JEHOVAH}

Initialement écrit pour le calcul de l'écoulement d'un jet diphasique, le code Jehovah a été sujet à des améliorations de façon à étendre son champ d'application. Nous présentons dans ce qui suit les différentes versions du code qui permettent le calcul dans diverses géométries :

- géométrie annulaire : calcul de l'écoulement dans le downcomer d'un réacteur à eau sous pression en condition d'injection de secours
- géométrie axisymétrique : calcul d'un jet libre, confiné ou face à une plaque

- géométrie plane : calcul de l'écoulement au voisinage d'une jonction de deux tuyauteries (version "TÉ ")

\subsection{Version " DOWNCOMER"}

Cette version, décrite en détail dans (Werkoff, 1982), simule l'écoulement bidimensionnel dans le downcomer d'un réacteur à eau sous pression en condition d'injection de secours. La figure $I$ illustre une image du comportement du réacteur dans ces conditions. En effet, on est dans la situation où une grosse brèche en branche froide s'est produite; le cœur est totalement dénoyé et de l'eau froide est injectée dans les branches froides. Cette eau doit descendre vers le fond de la cuve à travers le downcomer afin de faire monter le niveau dans le cuve et ainsi renoyer le cœur. La descente d'eau dans le downcomer est limitée par l'effet de by-pass: l'eau injectée dans les branches intactes peut en partie transiter vers la branche rompue. Ce phénomène est dû à l'effet d'entraînement de la vapeur venant du cœur et remontant dans le downcomer.

\subsubsection{Présentation de la géométrie}

Le domaine bidimensionnel utilisé (figure 2) est rectangulaire et représente la développée du downcomer où l'on a supposé que $\delta$, l'épaisseur de l'anneau formant le downcomer, est très inférieure à toutes les autres dimensions du système. On néglige aussi les variations des paramètres suivant $y$. Le système de coordonnées choisi est rectangulaire $(x, z)$, le maillage également. En conséquence, les canalisations (ou branches) froides ainsi que les branches chaudes (obstacles) sont représentées par des rectangles. Le fond de cuve est représenté par un domaine rectangulaire de section $S$ choisie de telle sorte que $S \times \delta$ représente le volume de fond de cuve.

Ce domaine rectangulaire doit satisfaire à des conditions de périodicité pour l'ensemble des variables $X: X(x=0)=X(x=2 \pi R)$. Toutefois si le flux de vapeur entrant dans le fond de cuve est réparti uniformément et que par chaque branche froide est injectée la même quantité d'eau, le problème est alors symétrique et il est suffisant de ne traiter qu'un espace réduit de moitié avec des conditions de symétrie en $x=0$ et $x=\pi R$ (figure 3).

\subsubsection{Conditions aux limites}

Sur les parois, c'est-à-dire en bas et en haut du downcomer ainsi que sur les parois des branches chaudes qui sont représentées par des obstacles, on impose les vitesses normales nulles pour chacune des phases.

Pour les branches froides intactes et le fond de cuve, on ajoute localement, mais de façon uniforme sur la surface concernée, des termes sources $J_{\text {inj }}$ dont on impose la valeur et qui interviennent aux seconds membres des équations de masse. Des termes correspondants $J_{\text {inj }} u$ et $J_{\text {inj }} w$ sont ajoutés aux seconds membres des équations de moment. Enfin aux seconds membres des équations d'énergie on ajoute des termes $J_{\text {inj }}\left(h_{\mathrm{inj}}-h\right)$ où $h_{\mathrm{inj}}$ est l'enthalpie spécifique imposée du fluide injecté. 


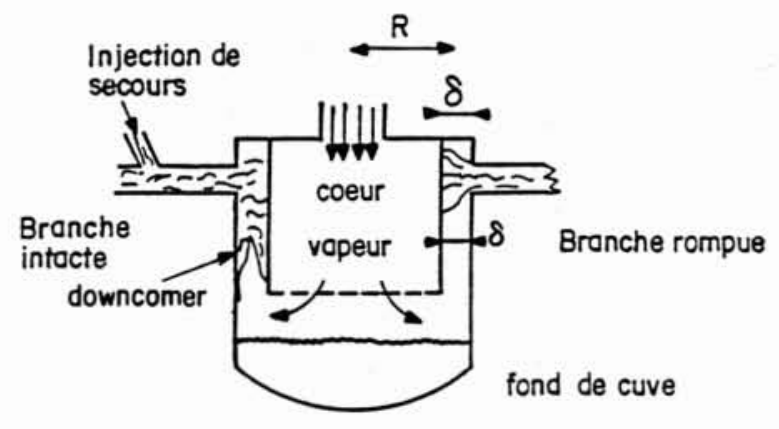

Figure 1 - Schéma de principe du Down-Comer.

Figure 2 - Projection en 2D d'un Down-Comer. Cas d'un réacteur 4 boucles.

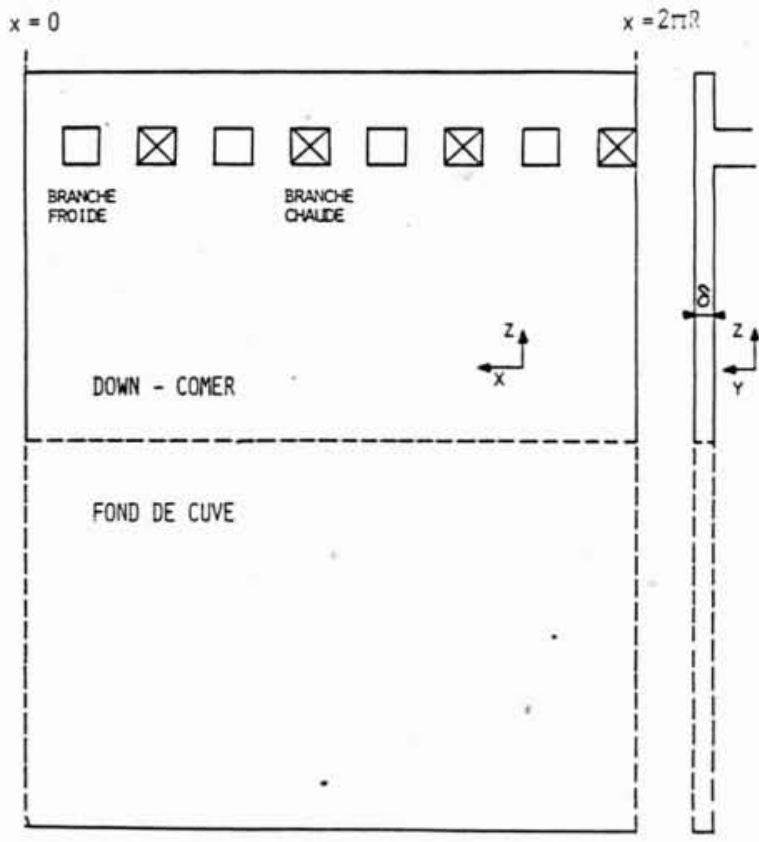

Cette version est écrite pour traiter des écoulements axisymétriques dans le sens Sud-Nord et Est, c'est-à-dire que le fluide est rentrant en Sud et sortant en Nord et/ou en Est.

Les conditions aux limites utilisées sont :

- En Sud :

- la pression, les enthalpies, le taux de présence et les vitesses radiales (nulles car écoulement monodimensionnel) imposés, ou

- la condition de fond borgne : vitesses axiales imposées nulles.

- En Nord ou Est :

- la pression imposée uniquement (écoulement sortant), ou

- la condition de paroi : vitesses normales nulles.

On a alors la possibilité d'effectuer des calculs dans les géométries suivantes ( figure 7 ) :

- jet libre,

- jet confiné (élargissement brusque);

- jet face à une plaque.

L'option fond borgne permettant de calculer des décompressions de capacité dans les géométries ci-dessus.

Les figures 8,9 et 10 illustrent des résultats de calcul de reconstitution d'expériences dans les 3 configurations. Le calcul du jet libre (reconstitution de l'expérience Jericho; Gully, 1979) fait apparaittre une différence de comportement entre le liquide et la vapeur. En effet, on constate sur la figure 8.1 que la vapeur est beaucoup plus déviée que le liquide qui a tendance à ne pas changer de direction. Par ailleurs, on observe une bonne prédiction du champ de pression ( figure 8.2) avec cependant une légère sous estimation. 


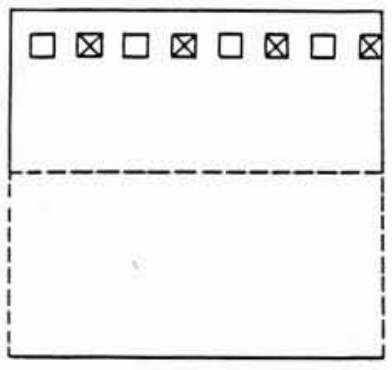

$\square \otimes \square \otimes \square \otimes$

PERIODICITE

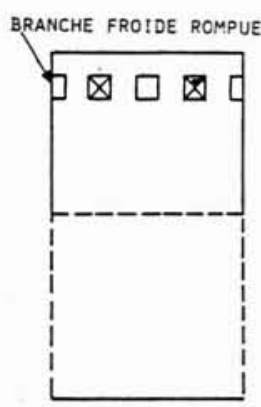

BOUCLES

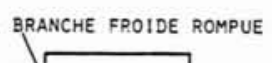

$\nabla \square \square$

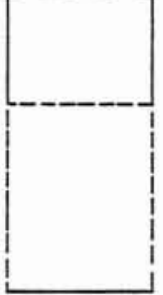

3 BOUCLES

(1)

SYMETRIE

(1/2 DOWN - COMER)

Figure 3 - Configurations possibles.

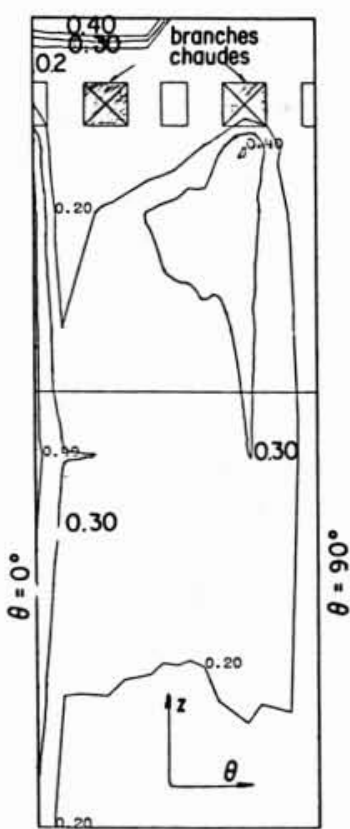

iso- toux de vide

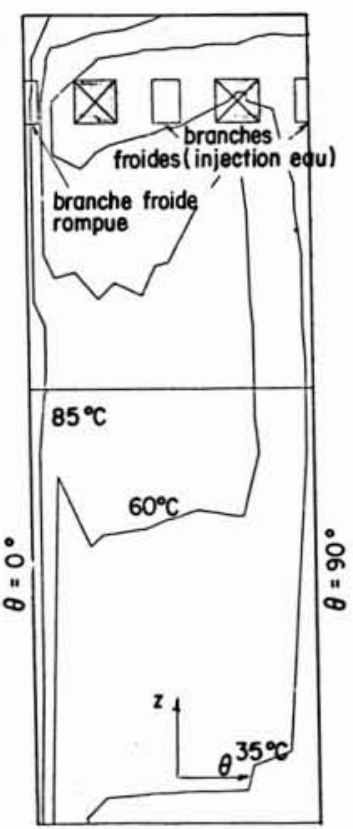

isothermes

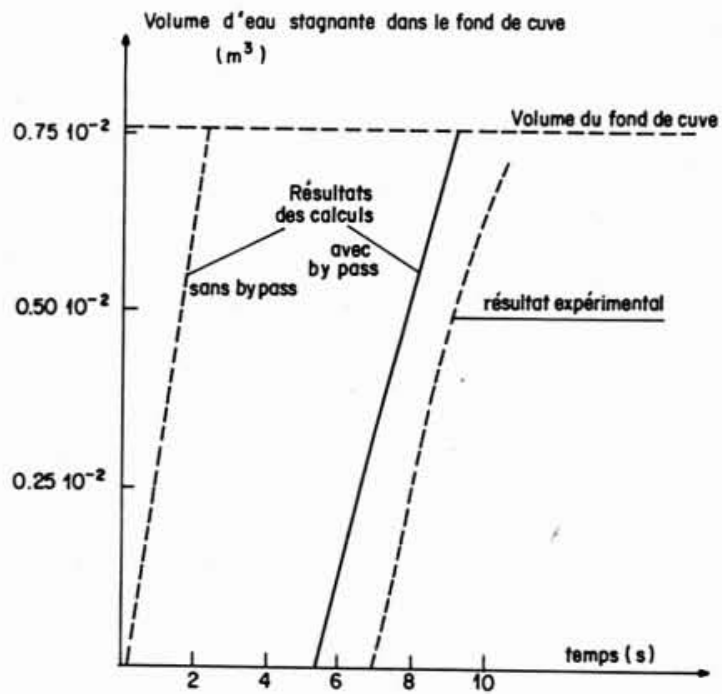

Figure 5

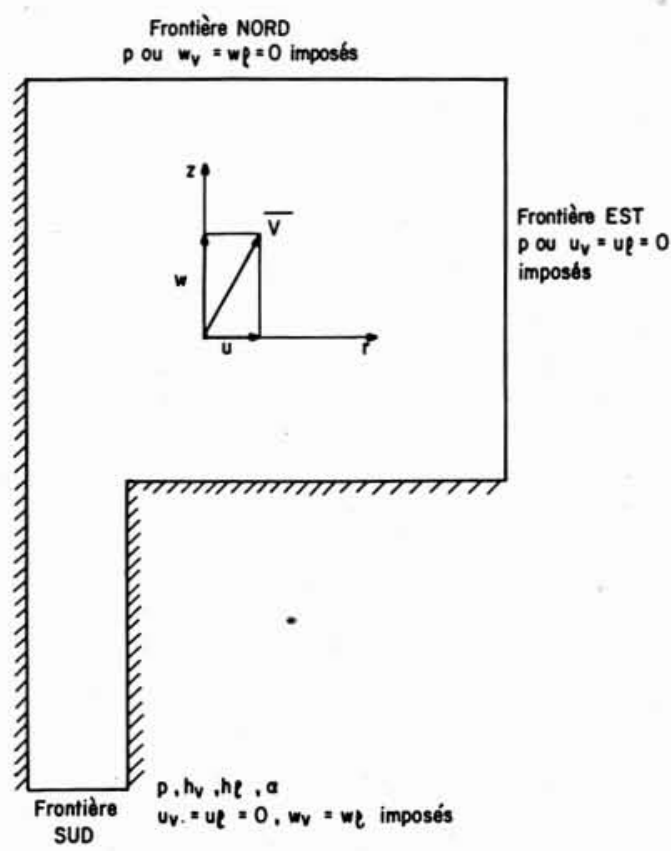

Figure 6 - JEHOVAH version " jet ".

Domaine de calcul.

Figure 4 - JEHOVAH version "downcomer ".

Domaine de calcul.

Résultats de calcul (expérience CREARE). 

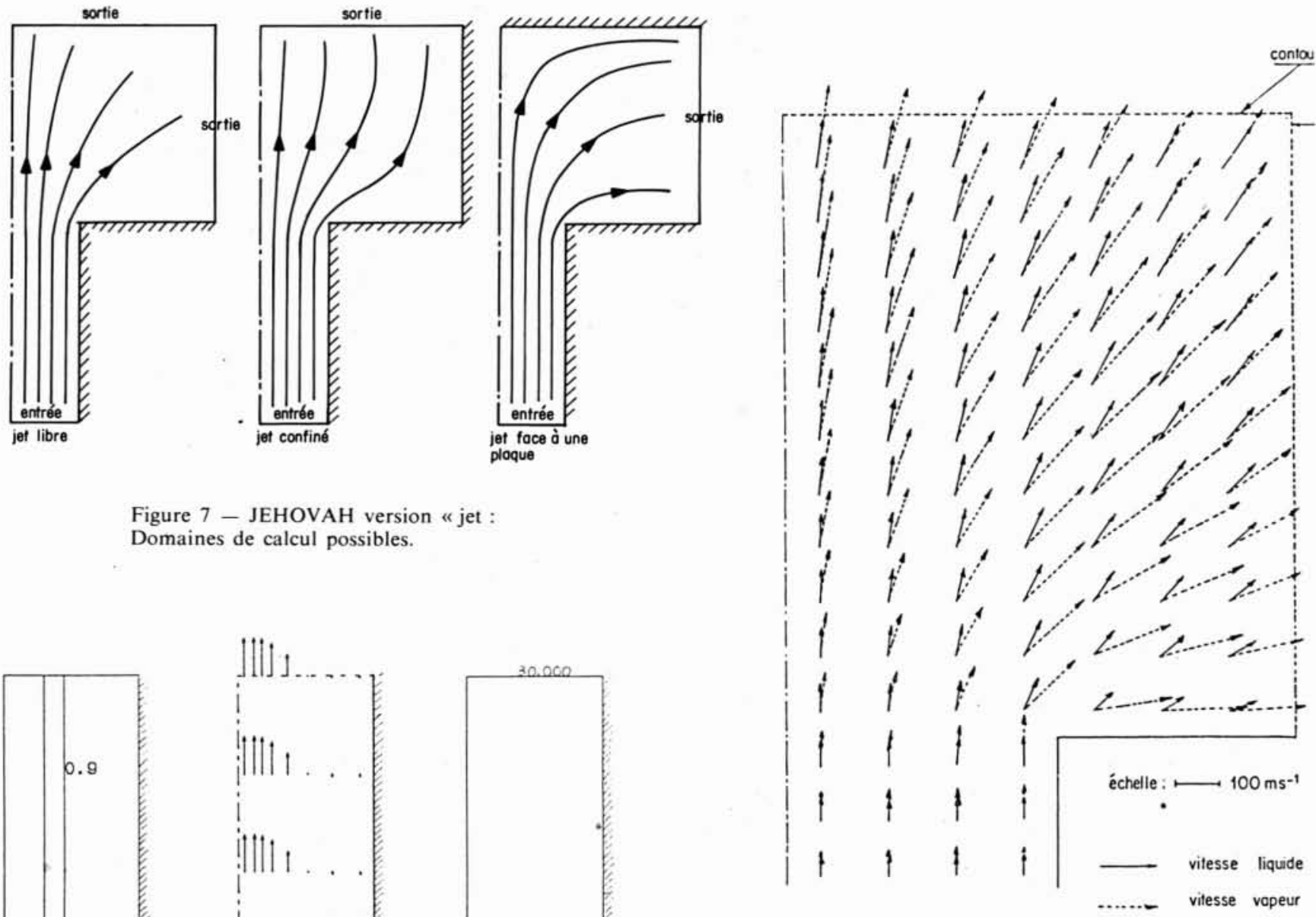

Figure 7 - JEHOVAH version « jet :

Domaines de calcul possibles.
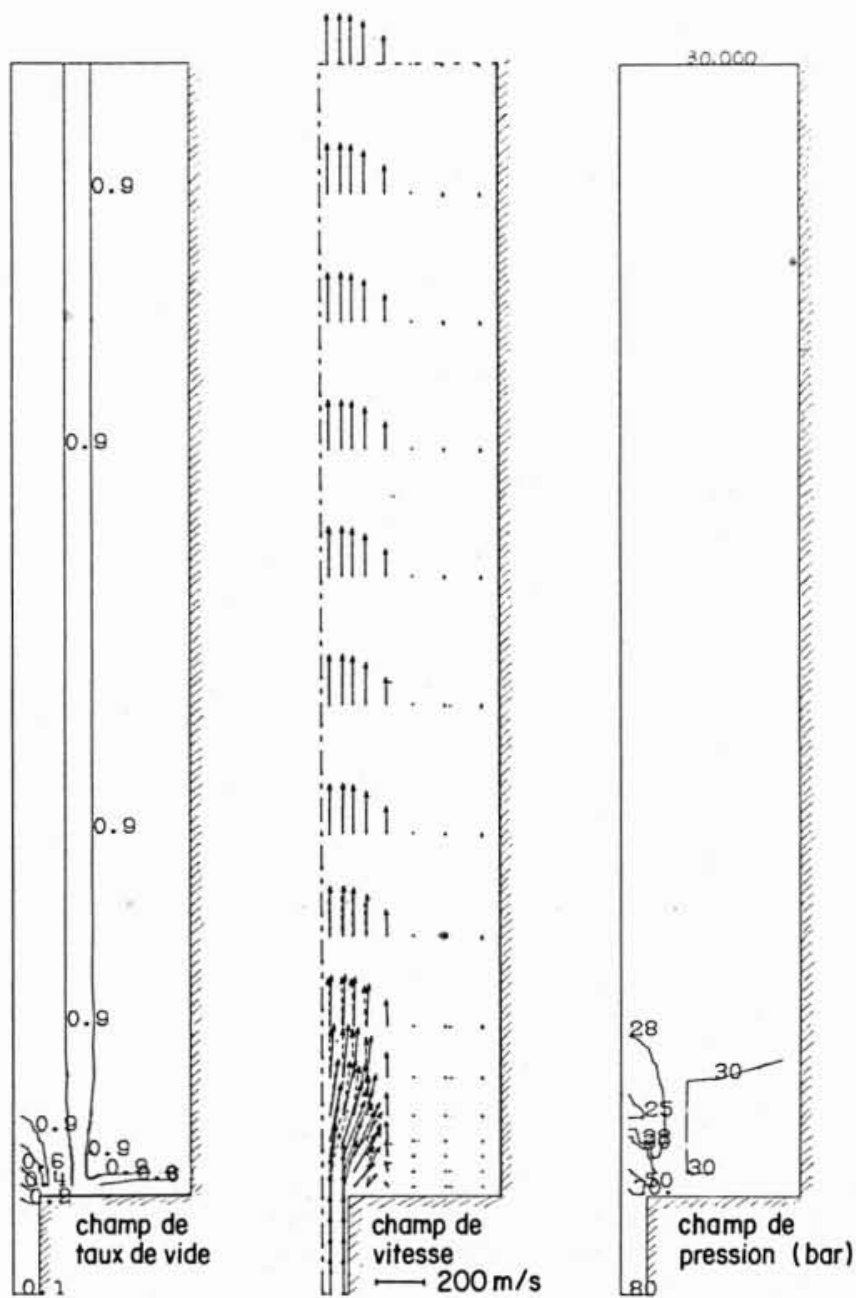

Figure 8.1 - Champ de vitesse des deux phases calculées.

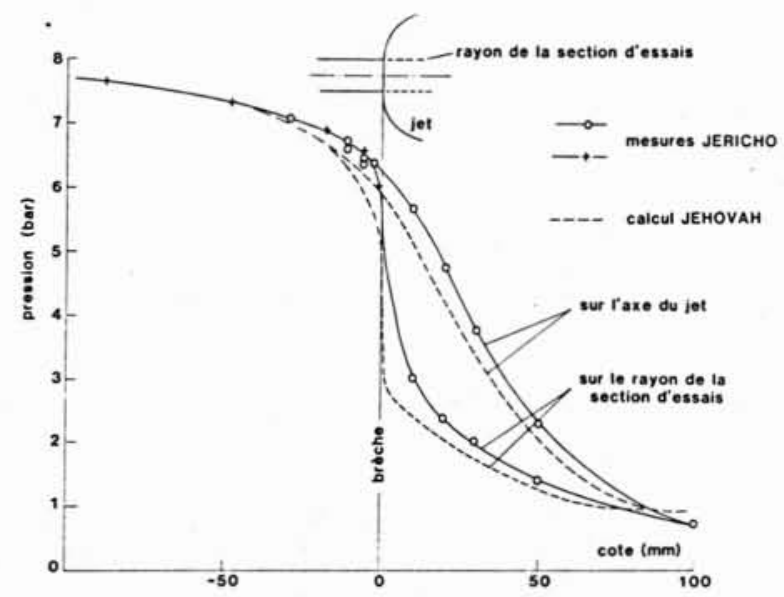

Figure 9 - Calculs JEHOVAH en configuration de jet confiné (expérience Super Moby Dick).

Cas $\alpha=0,1$ à l'entrée.

Figure 8.2 - Profils de pression mesurée (JERICHO) et calculée (JEHOVAH). 

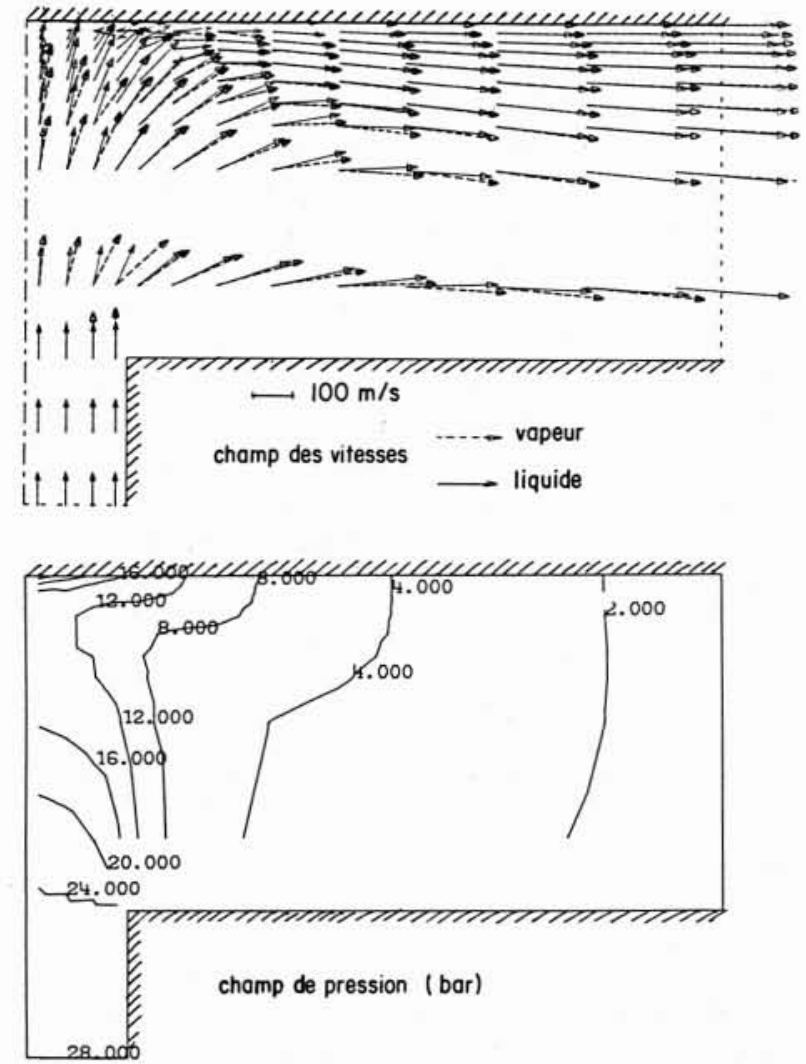

Figure 10 - Ecoulement d'un jet face à une plaque (Expérience HDR 3000)

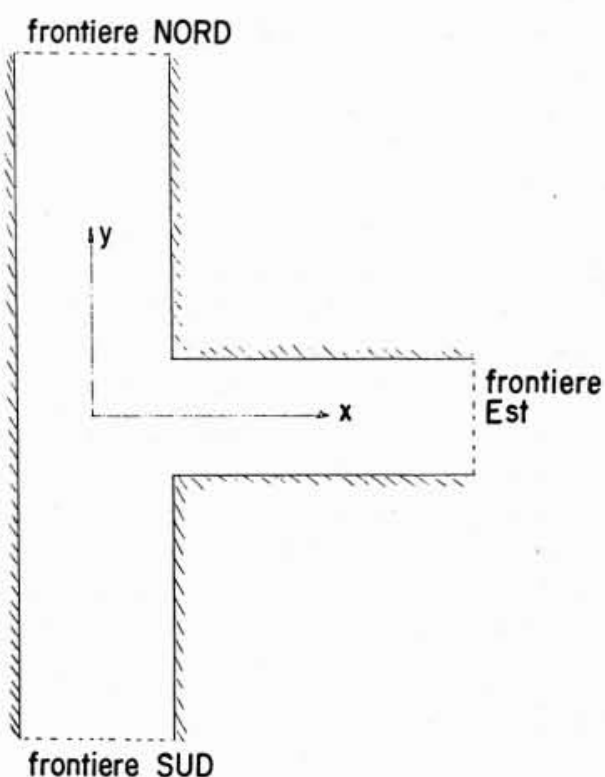

Figure 11 - Version "Te». Domaine de calcul.

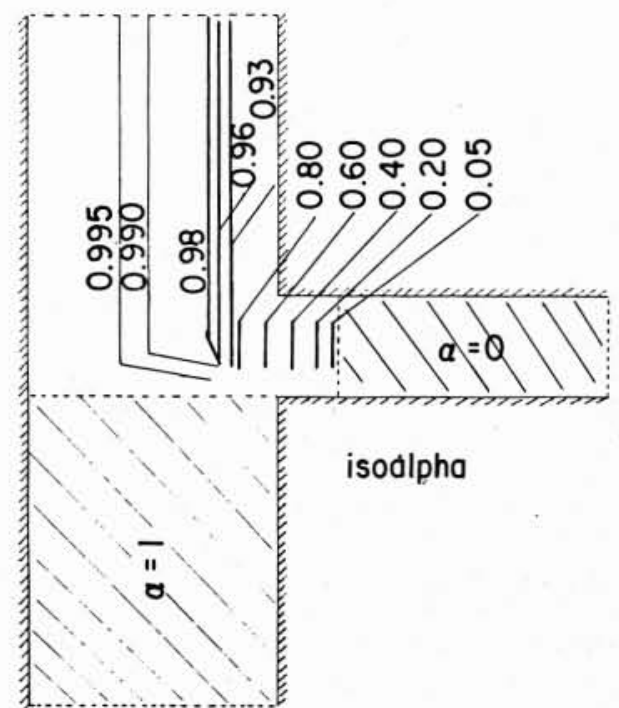

Figure 12 - Résultats de calcul d'un " Te ". (Expérience EPIS II, condensation partielle).
Le calcul du jet confiné (figure 9) fait ressortir la présence de profils radiaux de vitesse, profils observés expérimentalement (Jeandey, 1983), ainsi qu'une détente du fluide diphasique quelques diamètres en aval de l'élargissement brusque.

Enfin, la figure 10 montre les résultats de calcul de l'écoulement d'un jet face à une plaque. Les isobarres particulièrement intéressantes font apparaitre une zone de recompression autour de l'axe.

\subsection{Version " $T E$ "}

Cette version permet le calcul de l'écoulement au voisinage d'une jonction de 2 tuyauteries. Cette jonction est représentée par un "té " : bidimensionnel en $x, y$ (figure 11). Ayant conscience que ce problème est de toute évidence tridimensionnel, c'est une schématisation à l'échelle d'un plan, les variations suivant $z$ étant supposées négligeables.

La géométrie se compose d'une branche principale orientée Sud-Nord à laquelle est reliée perpendiculairement une branche intermédiaire. Cet ensemble est orientable par rapport à la verticale, c'est-à-dire que les forces de 
gravité projetées sur les axes $x$ et $y$ sont prises en compte dans les équations de moment.

La condition initiale du calcul est un régime uniforme au repos. Le transitoire de calcul résulte de la variation des conditions aux limites.

A ce sujet, on impose aux frontières Sud, Nord et Est ( figure 11):

- si l'écoulement est rentrant, les vitesses normales (débit imposé), les enthalpies, le taux de présence ainsi que les vitesses transversales (nulles car écoulement monodimensionnel);

- si l'écoulement est sortant, la pression uniquement.

Pour cette version, on ne traite que le cas des écoulements co-courants aux frontières.

Les parois sont traitées de la même façon que pour les autres versions. Sur la figure 12 sont portés le champ de vitesse et les iso-alpha obtenus lors d'un calcul de reconstitution de l'expérience EPIS II en condensation partielle.

On y remarquera la présence d'un fort gradient de $\alpha$ au voisinage de la jonction.
3. Conclusions

Le code Jehovah est issu d'un modèle bidimensionnel à deux fluides.

La discrétisation est faite à l'aide d'une méthode semi-implicite à mailles décalées. Le maillage rectangulaire est variable. A l'heure actuelle, ce code permet le calcul des écoulements diphasiques bidimensionnels dans des géométries diverses à l'aide de 3 versions de ce code :

- version "downcomer " : écoulement dans le downcomer d'un réacteur à eau sous pression en début d'injection de secours avec simulation des branches chaudes et froides;

- version " jet " : écoulement d'un jet libre, confiné ou face à une plaque;

- version "Té " : écoulement au voisinage d'une jonction de deux tuyauteries.

Des résultats de calculs dans ces géométries sont présentés et démontrent l'aptitude du code Jehovah à traiter des cas d'écoulement diphasique bidimensionnel dans des géométries complexes.

\section{Références}

BERNARD J., CHAULIAC C., 1981. - Programme EPIS 2 Rapport final, Rapport EMT/SMTS/TTMF/81-25 (rapport interne).

BROADUS C.R., JAMES S.W., LEE W.H., LIME J.F., PATE R.A., 1977. - Becon/Mod $2-A C D C 7600$ Computer program for analyzing the flow of mixe air, steam and water in a containment system, Idaho National Engineering Laboratory, Department of Energy, CDAP-TR-002.

DELHAYE J.M., 1970. - Contribution à l'étude des écoulements diphasiques eau-air et eau-vapeur, Thèse Docteur ès Sciences, Faculté des Sciences, Université de Grenoble.

DELHAYE J.M., ACHARD J.L., 1977. - On the use of averaging operators in two-phase flow modeling, Thermal and Hydraulic Aspects of Nuclear Reactor Safety, Vol. 1, Light Water Reactors, JONES O.C. \& BANKOFF S.G., Eds, ASM, pp. 289-332.

DELHAYE J.M., ACHARD J.L., 1978. - On the averaging operators introduced in two-phase flow modeling. Transient
Two-Phase Flow, Proceedings of the CSNI Specialists Meeting, August 3-4, 1976, Toronto, BANERJEE S. \& WEAVER K.R., Eds, AECL, Vol. 1, pp. 5-84.

GULLY Ph., 1979. - Jet diphasique eau-vapeur, Thèse Docteur-Ingénieur, Université Scientifique et Médicale de Grenoble:

JEANDEY Ch., GROS D'AILLON L., BARRIERE G., BOURGINE R., VERDUN C., 1983. - Mesure de débits critiques sur l'installation Super Moby Dick dans une section d'essais comportant un divergent ou un élargissement brusque, Note T/SETRE/82-32 (rapport interne).

MAZILLE J.E., 1979. - Jehovah - Programme de calcul d'écoulements diphasiques transitoires bidimensionnels à partir d'un modèle à fluides séparés, Note TT n ${ }^{\circ} 624$ (rapport interne).

WERKOFF F., 1982. - Etudes numériques des écoulements diphasiques dans des géométries downcomer, Note TT/SETRE/82-6 (rapport interne). 
Ph. GULLY et F. WERKOFF

237

Annexe

Système d'équations du modèle JEHOVAH

$\underline{\text { MASSE }} \quad\left[\begin{array}{l}\frac{\partial}{\partial t} \alpha \rho_{v}+\frac{1}{r} \frac{\partial}{\partial r} r \alpha \rho_{v} u_{v}+\frac{\partial}{\partial z} \alpha \rho_{v} w_{v}=\Gamma \\ \frac{\partial}{\partial t}(1-\alpha) \rho_{\ell}+\frac{1}{r} \frac{\partial}{\partial r} r(1-\alpha) \rho_{\ell} u_{\ell}+\frac{\partial}{\partial z}(1-\alpha) \rho_{\ell} w_{\ell}=-\Gamma\end{array}\right.$

$\frac{\text { QUANTITE DE }}{\text { MOUVEMENT SUR } r}\left[\begin{array}{l}\alpha \rho_{v}\left(\frac{\partial}{\partial t} u_{v}+u_{v} \frac{\partial}{\partial r} u_{v}+w_{v} \frac{\partial}{\partial z} u_{v}\right)+\alpha \frac{\partial}{\partial r} p=M_{r}-u_{v} r \\ (1-\alpha) \rho_{\ell}\left(\frac{\partial}{\partial t} u_{\ell}+u_{\ell} \frac{\partial}{\partial r} u_{\ell}+w_{\ell} \frac{\partial}{\partial z} w_{\ell}\right)+(1-\alpha) \frac{\partial}{\partial r} p=-M_{r}+u_{\ell} r\end{array}\right.$

$\frac{\text { QUANTITE DE }}{\text { MUUVEMENT SUR } z}=\left[\begin{array}{l}\alpha \rho_{v}\left(\frac{\partial}{\partial t} w_{v}+u_{v} \frac{\partial}{\partial r} w_{v}+w_{v} \frac{\partial}{\partial z} w_{v}\right)+\alpha \frac{\partial}{\partial z} p=M_{z}-w_{v} \Gamma+\alpha \rho_{v} g_{z} \\ (1-\alpha) \rho_{\ell}\left(\frac{\partial}{\partial t} w_{l}+u_{\ell} \frac{\partial}{\partial r} w_{\ell}+w_{\ell} \frac{\partial}{\partial z} w_{\ell}\right)+(1-\alpha) \frac{\partial}{\partial z} p=-M_{z}+w_{\ell} \Gamma+(1-\alpha) \rho_{\ell} g_{z}\end{array}\right.$

$\left[\begin{array}{l}\alpha \rho_{v}\left(\frac{\partial}{\partial t} h_{v}+u_{v} \frac{\partial}{\partial r} h_{v}+w_{v} \frac{\partial}{\partial z} h_{v}\right)-\alpha\left(\frac{\partial}{\partial t} p+u_{v} \frac{\partial}{\partial r} p+w_{v} \frac{\partial}{\partial z} p\right)=E-\Gamma\left(h_{v}+\frac{v_{v}^{2}}{2}\right) \\ -\left(M-\Gamma v_{v}\right) \cdot v_{v} \\ (1-\alpha) \rho_{\ell}\left(\frac{\partial}{\partial t} h_{\ell}+u_{\ell} \frac{\partial}{\partial r} h_{\ell}+w_{\ell} \frac{\partial}{\partial z} h_{\ell}\right)-(1-\alpha)\left(\frac{\partial}{\partial t} p+u_{\ell} \frac{\partial}{\partial r} p+w_{\ell} \frac{\partial}{\partial z} p\right)= \\ -E+\Gamma\left(h+\frac{v_{\ell}^{2}}{2}\right)+\left(M-\Gamma v_{\ell}\right) \cdot v_{\ell}\end{array}\right.$ 


\section{Discussion}

Président : M. F. BouLoT

\section{Le Président. - $\mathrm{Y}$ a-t-il des questions ?}

M. HufFenus. - La modélisation utilisée dans Jehovah est fondée sur les mêmes principes que celle développée dans CATHARE; c'est dire qu'elle est particulièrement bien adaptée à une simulation monodimensionnelle. Le fait de passer à 2 dimensions ne modifie-t-il pas un peu la structure de la modélisation en particulier pour les phénomènes agissant dans le sens transversal à l'écoulement?

Dans cette même optique, on devrait voir apparaître les termes de diffusion et de convection turbulente... à moins que l'on laisse à la diffusivité numérique le soin de représenter les phénomènes !

Labsence de ces termes dans Jehovah permet semble-t-il d'assimiler un calcul à taux de vide $\alpha=0$ ou $\alpha=1$ (monophasique) fait par ce programme à un calcul en fluide parfait avec une valeur empirique du frottement en paroi.

M. GULLY. - Il n'y a pas de terme de turbulence dans le modèle. Le calcul en simple phase ne doit pas être possible, il ne l'est qu'en diphasique avec transfert de masse.

M. RousSEAU. - On n'a jamais essayé d'utiliser ce mode en monophasique pour la raison qu'on a fait l'hypothèse que les termes principaux dans l'équation de moment radiale étaient justement les termes de transfert de masse.

Le Président. - Est-ce que les phénomènes de jets ont pu vraiment être traités ainsi ?

M. GULLY. - On a qualifié le code sur une expérience de jet diphasique comme vous l'avez-vu.

Le Président. - J'ai vu une courbe de pression.

M. GULLY, - Les pressions sont mesurées sur l'axe du jet et sur le rayon de la section d'essai. Le calcul des pressions est satisfaisant.

Le Président. - Il me semble me rappeler que, dans un jet, la pression n'est peut être pas la variable la plus sensible à la modélisation. Il y a des jets qui se calculent à pression constante.

M. GuLLY. - C'est un jet diphasique présentant un gradient radial de pression très important.

M. SCHмiтt. - Je voudrais revenir sur ces termes qui sont caractéristiques de la turbulence. Poser $\overline{u v}=\bar{u} \bar{v}$ revient à supprimer toute convection turbulente. Ainsi dans le cas d'une simulation d'un jet dans un espace infini ou non, le résultat du calcul montre que le jet va tout droit, sans mélange avec l'extérieur et avec dégazage depuis sa surface. On peut alors se demander si cette solution est réaliste et si, dans ce cas, les phénomènes de transferts turbulents ne sont pas prédominants. Personnellement, $j$ 'ai fait des expériences avec du fréon surchauffé que l'on injecte et voici ce que j'ai observé : le fréon éclate bien et remplit rapidement toute la veine.

M. GuLLY. - Y avait-il un noyau de liquide au centre du jet,
M. SСнмITt. - Il était difficile d'aller y voir...

M. MASBERNAT. - Dans la mesure où on néglige les effets turbulents dans le transfert de quantité de mouvement, on peut s'attendre à une bonne prédiction de la pression mais à une moins bonne prédiction des vitesses et des taux de vide. Cependant, si le transfert de quantité de mouvement aux interfaces est très important, il peut avoir un effet prédominant vis-à-vis des transferts turbulents en assurant un mélange de même type.

M. Gully. - Dans cette expérience, j’ai aussi effectué des mesures de $\rho V^{2}$ que je n'ai pas présentées. La concordance avec le calcul était assez bonne.

M. HUFFENUS. - L'interprétation de ces mesures en diphasique est-elle claire ?

M. GuLLY. - Jai mesuré un effort sur une cible locale placée en différents points du jet. La concordance avec le calcul étant assez bonne, les vitesses sont assez bien prédites par le code.

M. RIGAUDEAU. - Je voudrais savoir si vous avez l'intention d'introduire le calcul bidimensionnel dans CATHARE. Si vous passez par des taux de vide très fort ou très faible cela réduit beaucoup les possibilités d'application potentielle du code.

M. GULLY. - Il est possible que ce code bidimensionnel fasse partie, dans le futur, des modules CATHARE pour représenter le downcomer. Son introduction pose encore certains problèmes.

M. ROUSSEAU. - Je voudrais répondre d'une façon plus précise. CATHARE ne possède actuellement que le calcul monodimensionnel. Des éléments nouveaux sont envisagés pour une version future CATHARE II. En particulier, on envisage un calcul bidimensionnel pour le downcomer qui serait utilisé dans la phase de remplissage où peut intervenir le phénomène de bypass. vide?

M. RIGAUDEAU. - Vous n'avez pas de cas extrême de taux de

M. RousSeAU. - Non. La décision n'est pas encore prise de l'introduire; il se peut qu'on s'arrête à la solution du code uniquement monodimensionnel et que l'on cherche à rendre compte, par des procédés monodimensionnels, des effets bidimensionnels calculés par le code à 2 dimensions.

Le Président. - Y a-t-il d'autres remarques ou questions?

Puisqu'il n'y en a pas, nous allons arrêter là la discussion sur l'exposé de M. Gully.

Je crois que, demain, un autre exposé est prévu sur les écoulements à deux dimensions et on aura ainsi l'occasion de revenir sur ce problème.

Nous terminons cet après-midi en remerciant les orateurs des exposés particulièrement précis qu'ils ont faits et qui ont été très appréciés par l'auditoire.

La séance est levée à 17 h 30 . 